AperTO - Archivio Istituzionale Open Access dell'Università di Torino

\title{
Power Grid Outlier Treatment through Kalman Filter
}

\section{This is the author's manuscript}

Original Citation:

Availability:

This version is available http://hdl.handle.net/2318/158768

since

Publisher:

Lisa O'Conner

Terms of use:

Open Access

Anyone can freely access the full text of works made available as "Open Access". Works made available under a Creative Commons license can be used according to the terms and conditions of said license. Use of all other works requires consent of the right holder (author or publisher) if not exempted from copyright protection by the applicable law. 


\section{(3) \\ UNIVERSITÀ DEGLI STUDI DI TORINO}

This is an author version of the contribution published on:

Questa è la versione dell'autore dell'opera:

IEEE International Symposium on Software Reliability Engineering Workshops,

Naples, Italy, 407 - 412, 3-6 Nov, 2014

The definitive version is available at:

La versione definitiva è disponibile alla URL:

$h t t p: / / i e e x p l o r e . i e e . . o r g / x p l / l o g i n . j s p ? t p=\&$ arnumber=6983875\&url=http\%3A\%2F \%2Fieeexplore.ieee.org\%2Fiel7\%2F6982004\%2F6983760\%2F06983875.pdf\%3Farnu mber\%3D6983875 


\title{
Power Grid Outlier Treatment through Kalman Filter
}

\author{
Cesario Di Sarno*, Alessia Garofalo*, Gianfranco Cerullo* \\ Nadir Murru $^{\dagger}$, Felicita Di Giandomenico ${ }^{\dagger}$, Silvano Chiaradonna ${ }^{\dagger}$ \\ *University of Naples "Parthenope", Department of Engineering, Naples, Italy \\ email: \{cesario.disarno, alessia.garofalo,gianfranco.cerullo\}@uniparthenope.it \\ ${ }^{\dagger}$ CNR di Pisa - Istituto di Scienza e Tecnologie dell'Informazione (ISTI), \\ email: \{nadir.murru, felicita.digiandomenico, silvano.chiaradonna\}@isti.cnr.it
}

\begin{abstract}
Power grid monitoring is a very challenging task to avoid both disasters and economic damages. This is because many critical services e.g. health and welfare use the power grid as a reliable utility. Wide Area Monitoring System (WAMS) represents a technological solution to perform power grid monitoring. WAMS is based on two components: Phasor Measurement Unit (PMU) and Phasor Data Concentrator (PDC). PMUs are deployed within the power grid and their purpose is to provide synchronized measurements of both magnitude and angle phase of the electric signal. PDC analyses measurements provided by different PMUs to assess the global status of the power grid. In this paper we propose an enhanced WAMS architecture based on Kalman filter to improve state estimation and fault detection within the power grid; Kalman filter allows to reduce the noise effects on measurements gathered by PMUs. Experimental results show the effectiveness of the proposed solution.
\end{abstract}

Keywords-Power Grid, Wide Area Monitoring System, Kalman filter, Fault detection;

\section{INTRODUCTION}

Power Grid infrastructure, classified as Critical Infrastructure [1], is a very important asset of modern societies, used as a reliable utility by many important services (e.g., health and welfare). Thus, a failure of the power grid makes these essential services unavailable.

In last years, the power grid evolved to Smart Grid i.e., a power grid that uses the IT infrastructure to improve both energy efficiency on the power grid and energy distribution to the users' homes and offices. In particular, the smart grid uses two-way digital communication technology to exchange real-time information between devices deployed within the power grid, devices deployed within customers houses/offices and control centers [2]. Each device is considered as a sensor/actuator that can: gather data (power meters, voltage sensors, etc.) and send them to the control center by using LAN or WAN network; receive commands by the control center to perform specific control operations. The usage of both IT infrastructure and Internet network has increased the number of cyber-attacks the power grid is exposed to. According to the Symantec report [3], during the monitoring period from July 2012 to June 2013, nine attacks per day targeted the energy sector. In addition to cyberattacks, other factors as increasing load and consumption demands have enlarged electricity issues, such as blackouts, and overloads. For example in July 2012 for two days a large portion of the Indian power grid has experienced a blackout due to a $9 \%$ gap between the effective and the real available energy amount [4].

Thus, it is very important to monitor the power grid infrastructure to avoid disasters. For this purpose Wide Area Monitoring Systems (WAMSs) are used. In particular a WAMS makes use of two types of devices: Phasor Measurement Unit (PMU) and Phasor Data Concentrator (PDC). PMUs measure voltage and current phasor values in a specific area of power grid and send them to the PDC. PDC gathers phasors provided by different PMUs and analyzes them to evaluate the power grid status.

In this paper we propose an enhanced WAMS architecture to improve state estimation and fault detection within the power grid. The proposed architecture is based on a Kalman filter that performs real-time analysis of data gathered by PDC to detect anomalous power grid states called outliers. A simulator is used: i) to model the normal-abnormal behaviour of a real power grid, specifically a IEEE 9-bus system and ii) to generate a dataset containing the phasors values of each system bus at each time slot. The phasors contained within the dataset are equivalent to phasors generated by PMUs deployed at each bus of the IEEE 9-bus system. Additionally, the generated dataset contains a fault in one system bus. The dataset is analyzed through the Kalman filter for outlier detection. Experimental results show the effectiveness of the proposed solution.

The paper is organized as follows: in Section II an analysis on related works is presented; in Section III the enhanced WAMS architecture is discussed; in Section IV the IEEE9 bus system is described and details are provided about dataset generation; in Section $\mathrm{V}$ experimental results are shown and in Section VI conclusions are discussed.

\section{RELATED WORK}

State estimation of electric power grids to allow detection of faults has been addressed in the literature (an overview is in [5]).

Most of the classical studies of state estimator for electrical grids involve the weighted least-squares method (for 


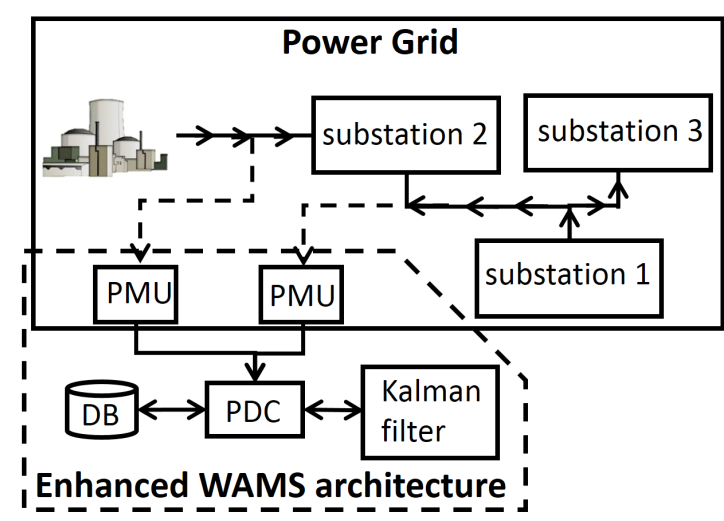

Figure 1: Enhanced Wide Area Monitoring System (WAMS) for power grid infrastructure

a survey see, e.g., [6]). Enlargement and increasing of complexity (principally due to penetration of renewable energies) in modern power grids have generated new scenarios of faults determining the necessity of further investigations in monitoring and treatment of outliers in state estimators.

In [7], a hierarchical state estimator based on the standard weighted-least squares method is developed only for detection of malicious false data injection. In [8] and [9] heuristic approaches are described and tested on IEEE-9 bus system.

Moreover, in recent years the development of PMUs for synchronized measurements have extended the scenarios for real-time monitoring of power grids. In [10], [11] a security analysis was performed about the vulnerabilities PMU are exposed to. Instead, in this paper we focus on techniques that allow to improve state estimation and fault detection within the power grid. Thus, we consider that PMU cannot be compromised.

In [12], a full weighted least square state estimator method combined with the use of PMUs has been proved on the IEEE-9 bus system. In [13] a new estimation method based on the unscentend Kalman filter has been studied. A twostage Kalman filtering technique has been developed in [14]. Since Kalman filter is not robust against outliers [15], these approaches can not provide good estimation when measurements are heavily affected by noise that produces bad measurements even in absence of faults.

In this paper, we propose a variant of the Kalman filter embedding an outlier detection feature, to make it appropriate to deal with measurements coming from PMUs, heavily affected by white noise.

\section{Proposed Architecture}

Figure 1 depicts our enhanced WAMS architecture for power grid monitoring. The solution proposed is based on Kalman filter that allows to detect faults within the power grid.

\section{A. Power Grid Description}

In Figure 1, the power grid (continuous rectangle) represents the data source that feeds the proposed architecture. A power system is usually divided into three parts: generation, transmission and distribution systems. In particular, the electricity is produced by converting mechanical energy into electrical energy.

The transmission system constitutes the back bone of power grid and it interconnects all generating stations to major load centers within the system. This system is organized as a meshed network that provides many alternative routes; this allows the power to flow from generators to loads.

The distribution system allows to transfer energy to the consumers. In particular, the consumers are connected to small generating plants near their own houses; these plants are connected to the distribution system.

\section{B. Enhanced Wide Area Monitoring System Architecture}

The enhanced WAMS architecture (dashed rectangle in Figure 1) is an infrastructure used to evaluate the realtime status of the power grid. The main idea of this approach is to analyze electric signal waveforms using phasors representation to monitor power grid behaviour. For example, if a power oscillation is detected then frequency, voltages and currents change from their nominal values. As a consequence, also the phasor changes from its own original value.

Two main components of the enhanced WAMS architecture are: Phasor Measurement Unit (PMU) and Phasor Data Concentrator (PDC) [16]. PMUs are hardware devices deployed on transmission lines and their purpose is to analyze the electric signal waveforms at different geographic locations at the same time. In particular each PMU samples the electric signal waveform provided by transmission line and generates the phasors. These phasors contain information about the status of the monitored transmission line in a specific moment and they are timestamped using the clock provided by the GPS receiver. These synchronized phasors are called synchrophasors. Timestamps can be used to compare collected synchrophasors to other PMUs with microsecond precision.

Phasor Data Concentrator (PDC) is a hardware/software component designed to: gather data provided by different PMUs; store gathered data in a database; perform a comparison between the synchrophasors to assess the status of power grid; exchange phasors with PDCs at different locations to perform wide area monitoring. In this work, Kalman filter is proposed to improve state estimation and fault detection performed by PDC. More details about Kalman filter are provided in the next subsection.

\section{Kalman filter with outlier treatment}

The Kalman filter is a Bayesian-based method for the state evaluation of dynamical systems subjected to white 
noise [17]. The white noise is a kind of noise that frequently affects signals and measurements and can be easily modeled with standard normal random variables.

To properly address fault detection, here we adopt a formulation of the Kalman filter embedding an outlier detection routine, based on a fuzzy algorithm developed in [18]. In the following, we describe our technique in general terms; its application to the electric context is shown in Section V.

The estimation of the measurand state at the step $k$ ( $0 \leq k \leq L$, discrete time with the maximum value $L$ determined by a stopping criterion) is denoted as a vectorial quantity $\mathbf{x}_{k}$. The measurement of the measurand is denoted by $\mathbf{z}_{k}$. In the following, we describe the algorithm only focusing on a generic component of the vectors $\mathbf{x}_{k}$ and $\mathbf{z}_{k}$ with the notation $x_{k}$ and $z_{k}$, respectively, for the sake of simplicity. At each measurement $z_{k}$ is associated the fixed uncertainty $\bar{\sigma}^{2}$ wich represents the inaccuracy of the measurement instrumentation. Similarly, $\sigma_{k}^{2}$ is the uncertainty associated to $x_{k}$ that is updated at each step $k$ by the following equation

$$
\sigma_{k}^{2}=\left(\frac{1}{\bar{\sigma}^{2}}+\frac{1}{\sigma_{k-1}^{2}}\right)^{-1}, \quad 0 \leq k \leq L .
$$

The starting $\sigma_{-1}^{2}$ is the given uncertainty associated to the pre-process estimation of the measurands $x_{0}^{-}=x_{-1}$ initialized from an expert judgment. The uncertainties $\sigma_{-1}^{2}$ and $\bar{\sigma}^{2}$ are initialized according to an expert judgment or based on technical specifications. The estimation of the measurand state at the step $k$ is determined by the following equation

$$
x_{k}=\frac{\bar{\sigma}^{2} x_{k}^{-}+\sigma_{k-1}^{2} z_{k}}{\bar{\sigma}^{2}+\sigma_{k-1}^{2}}, \quad 0 \leq k \leq L
$$

with

$$
x_{k}^{-}=x_{k-1}, \quad 1 \leq k \leq L .
$$

The previous equations are derived from [19] by modeling prior estimations and measurements as normal random variables. In particular measurements are modeled by the normal random variable $\mathcal{N}\left(z_{k}, \bar{\sigma}^{2}\right)$ and prior estimations by $\mathcal{N}\left(x_{k}^{-}, \sigma_{k-1}^{2}\right)$. In this way, the posterior estimation is modeled like a normal random variable obtained by the product of $\mathcal{N}\left(z_{k}, \bar{\sigma}^{2}\right)$ and $\mathcal{N}\left(x_{k}^{-}, \sigma_{k-1}^{2}\right)$. The result is still a normal random variable whose variance and mean are like in Eqs. (1) and (2). This is a straightforward application of the Bayes theorem in case of normal random variables. Finally, we use Eq. (3) for the update of the prior estimations, since in the next simulations the behavior of the measurand is unknown in the time evolution. Thus, we set the prior estimation at the step $k$ equal to the estimation at the step $k-1$. This is a standard choice in such a situation [17].

The solution adopted in the present work modifies Eq. (2) in order to treat the presence of possible outliers. In particular, following [18], we consider $z_{k}$ an outlier measurement

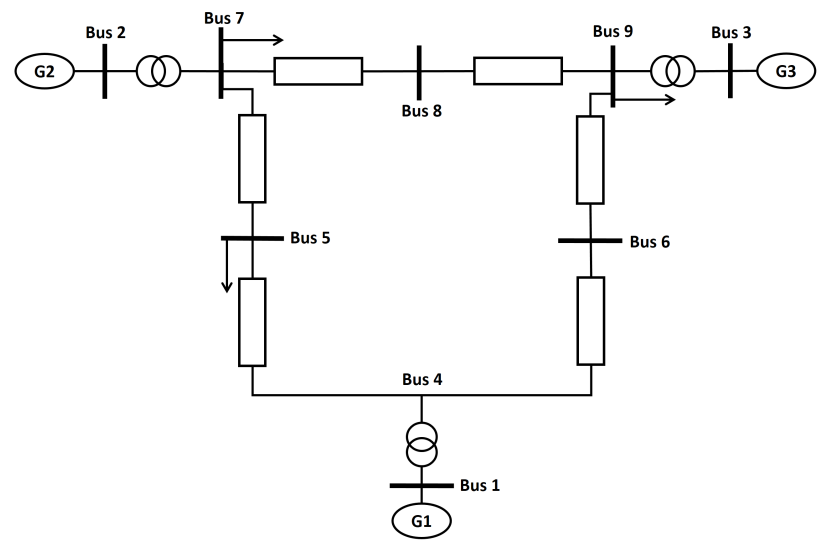

Figure 2: IEEE 9-bus system

when $\left|z_{k}-x_{k}^{-}\right|>2 \bar{\sigma}$ and we treat it introducing a weight $w$ defined as follows:

$$
\left\{\begin{array}{l}
w\left(z_{k}\right)=1 \quad \text { if } \quad\left|z_{k}-x_{k}^{-}\right| \geq 5 \bar{\sigma} \\
0<w\left(z_{k}\right)<1 \quad \text { if } 2 \bar{\sigma}<\left|z_{k}-x_{k}^{-}\right|<5 \bar{\sigma} \\
w\left(z_{k}\right)=0 \quad \text { otherwise }
\end{array}\right.
$$

when $2 \bar{\sigma}<\left|z_{k}-x_{k}^{-}\right|<5 \bar{\sigma}$ we evaluate $w\left(z_{k}\right)$ according to some fuzzy rules specified in [18]. The previous conditions are based on the three-sigma rule. Indeed, it is wellknown that a sampled datum from a normal distribution with mean $\mu$ and standard deviation $\sigma$ belongs to the interval $[\mu-3 \sigma, \mu+3 \sigma]$ with 0.99 probability. Thus, if $\left|z_{k}-x_{k}\right|<2 \bar{\sigma}$, we do not consider $z_{k}$ as an outlier measurement, since it is not far from the prior estimation. On the other hand, if $\left|z_{k}-x_{k}\right|>5 \bar{\sigma}$, the measurement $z_{k}$ can be considered an outlier measurement. Otherwise, the distance between measurement and prior estimation is not significant (neither too big nor too low) and a fuzzy treatment is preferable.

Thus, we change Eq. (2) using $w$ in order to properly manage outliers:

$$
x_{k}=\frac{\bar{\sigma}^{2} x_{k}^{-}+\sigma_{k-1}^{2} w\left(z_{k}\right) z_{k}}{\bar{\sigma}^{2}+\sigma_{k-1}^{2} w\left(z_{k}\right)}, \quad 0 \leq k \leq L
$$

In this way, the measurement $z_{k}$ is balanced by the weight $w$ so that outlier measurements do not affect the Kalman filter estimation process. Indeed, when $0 \leq w\left(z_{k}\right)<1$ the impact of the measurement $z_{k}$ is reduced in Eq. (5), since $\sigma_{k-1}^{2} w\left(z_{k}\right)<\sigma_{k-1}^{2}$.

\section{Scenario and Dataset Description}

In this section we describe the scenario used to validate the architecture proposed in the previous section. We used the IEEE 9-bus system (shown in Figure 2) which represents a portion of the Western System Coordinating Council (WSCC) 3-Machines 9-Bus system. Basically, this system contains 3 generators, 9 buses and 3 loads [20]. A simulator called MATPOWER [21] allows to simulate the IEEE 9bus system behaviour shown in Figure 2. In particular 
MATPOWER is a Matlab tool that allows to solve power flow and optimal power flow problems.

The system to be simulated e.g., IEEE 9-bus system is defined by a Matlab struct. The fields of this struct are "baseMVA", "bus", "branch", "gen". The "baseMVA" field is a scalar value while other fields are matrix. The "baseMVA" field specifies the system MVA base used for converting power into "per unit quantities". The "bus" data includes information about the voltage magnitude, voltage angle, total power generation and load at each bus. The "branch" data describes the flows and losses in each branch. The "gen" data allows to control the configuration parameters of each generator e.g., real and reactive power injections. Thus, different power grid dynamics can be simulated changing these parameters and running MATPOWER tool. The configuration parameters used to simulate the power grid shown in Figure 2 are detailed in Table I. The first six rows describe the configuration of three generators in terms of output power injected within the power grid, while the remaining rows describe the configuration of loads in terms of demanded power. In the configuration described: bus 1 is the reference bus, buses 2-3 are PV buses and remaining buses are PQ buses. Also both shunt conductance and susceptance values are equal to one for all buses.

In this study we focus on the analysis of phasors generated by different PMUs to detect e.g., a fault within power grid. Within "bus" matrix, MATPOWER provides information about the voltage magnitude and angle for each bus of simulated system. Thus we use these information as the phasors generated by PMUs. A single execution of MATPOWER outputs a single snapshot about the status of power grid according to specified inputs. MatDyn [22] is an open source tool used to perform dynamic analysis of electric power systems. It exploits MATPOWER engine to solve power flow problems. A new dataset was generated (with MatDyn) which contains the phasor values of each bus ( 9 in our case as shown in Figure 2) at each row. Thus, each dataset row represents a snapshot of power grid status per time unit. The frequency of phasors generation is $50 \mathrm{~Hz}$ (in compliance to the IEEE C37.118-2011 standard) and the time simulation chosen is 80 minutes. By using this configuration the dataset contains 240000 samples.

Also, the dataset contains a fault that is injected within the simulated power grid after 56 minutes. In particular a three-phase bus fault is simulated by increasing the shunt susceptance of the bus number 2 of about $e^{10}$, leading the voltage phasor measured at that bus to $\approx 0+j 0$. Of course the fault injected at bus number 2 affects the remaining part of the power grid, specifically the phasors measured at each bus.

\section{EXPERIMENTAL RESULTS}

In these simulations, we use the Kalman filter in order to determine the state of the IEEE 9-bus system, analyzing
Table I: Power Grid configuration through MATPOWER tool

\begin{tabular}{|c|c|c|c|}
\hline bus & Parameter & Value & Description \\
\hline 1 & $P_{G 1}$ & 71.95 & real power output (MW) \\
\hline 1 & $P_{\text {Max } 1}, P_{\text {Min } 1}$ & 250,10 & $\begin{array}{l}\text { maximum and minumum real } \\
\text { power output }(\mathrm{MW})\end{array}$ \\
\hline 2 & $P_{G 2}$ & 163 & real power output (MW) \\
\hline 2 & $P_{\operatorname{Max} 2}, P_{\text {Min } 2}$ & 300,10 & $\begin{array}{l}\text { maximum and minumum real } \\
\text { power output (MW) }\end{array}$ \\
\hline 3 & $P_{G 3}$ & 85 & real power output (MW) \\
\hline 3 & $P_{M a x 3}, P_{M i n 3}$ & 270,10 & $\begin{array}{l}\text { maximum and minumum real } \\
\text { power output (MW) }\end{array}$ \\
\hline 5 & $P_{D 5}$ & 90 & real power demand (MW) \\
\hline 5 & $Q_{D 5}$ & 30 & reactive power demand (MVAr) \\
\hline 7 & $P_{D 7}$ & 100 & real power demand (MW) \\
\hline 7 & $Q_{D 7}$ & 35 & reactive power demand (MVAr) \\
\hline 9 & $P_{D 9}$ & 125 & real power demand (MW) \\
\hline 9 & $Q_{D 9}$ & 50 & reactive power demand (MVAr) \\
\hline
\end{tabular}

the phasors determined by the PMUs in Section IV. Prior estimations are set as in Table II and we use the following uncertainties:

$$
\bar{\sigma}^{2}=0.5, \quad \sigma_{-1}^{2}=0.1
$$

for each component of the measurand vector which is composed by real and imaginary part of voltages for each bus (i.e., it has 18 components). The uncertainty $\bar{\sigma}^{2}=0.5$ related to measurements has been chosen greater than the uncertainty $\sigma_{-1}^{2}$ related to prior estimations, since in our scenario measurements are heavily affected by white noise. Simulations have been performed with MATLAB and we show the results in Figures 3, 4, 5, 6.

In Figures 3 and 4 the measurements (solid line) and the estimations (dot line), provided by the technique described in Section III-C, are shown for voltage real and imaginary part of bus 1 . In the figures, the circle on the y-axis is the prior estimation. Similarly, Figures 5 and 6 show the same quantities for bus 2 . The remaining buses show behaviors similar to bus 1 and are here omitted.

First of all, we can observe that our technique provides estimations without the heavy noise that affects the measurements. Indeed, before the fault (simulated from the step 168100) fluctuations of the voltage estimations are reduced with respect to measurements. Estimations amount between 0.5 and 1 for the voltage real part of bus 1 and around 0 for the voltage imaginary part of bus 1 , while measurements show wider oscillations between 1 and -1 in all the cases. Similar behavior for the remaining buses.

When the fault is injected within bus 2 (at step 168100) both real and imaginary parts of this bus drop to 0 after few seconds (as shown in Figure 5 and 6). Figures 3 and 4 show the effects of this fault on the bus 1. In particular, we can observe that the estimations of the imaginary part of voltage take a constant behavior instead of their characteristic fluctuations. Similarly, the estimations of the real part of voltage take a monotonic decreasing.

Thus, in this preliminary work we highlighted that Kalman filter dynamic can be used to perform the fault 
Table II: Prior estimation of bus voltages

\begin{tabular}{lll}
\hline Bus & Voltage real part est. & Voltage imaginary part est \\
\hline 1 & 1 & 0 \\
2 & 0.9858 & 0.1679 \\
3 & 0.9965 & 0.0832 \\
4 & 0.9861 & -0.0414 \\
5 & 0.9731 & -0.0683 \\
6 & 1.0028 & 0.0337 \\
7 & 0.9856 & 0.0107 \\
8 & 0.9940 & 0.0660 \\
9 & 0.9549 & -0.0726 \\
\hline
\end{tabular}

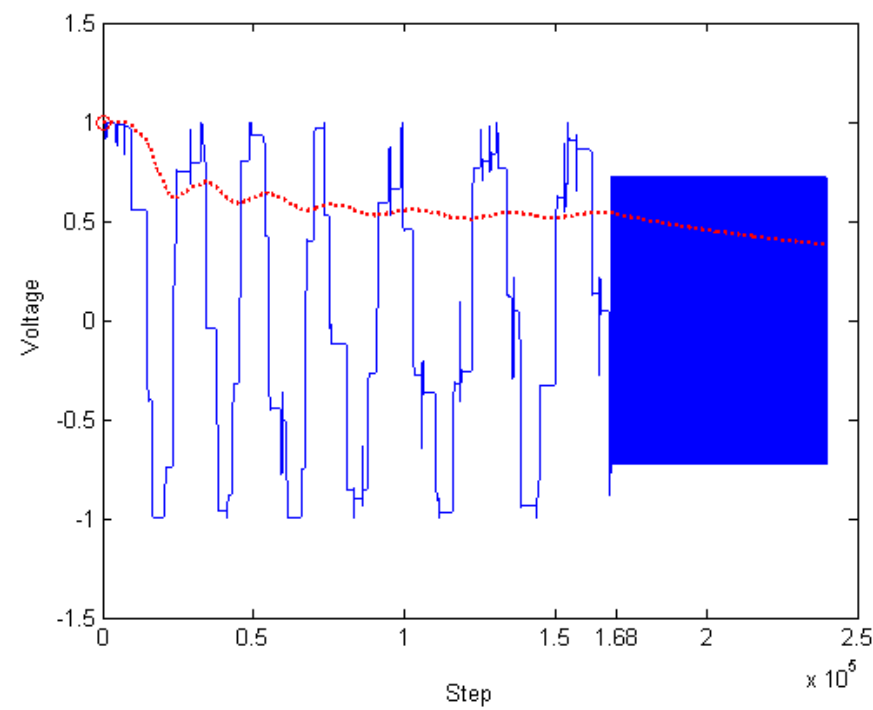

Figure 3: Measurements (solid line) and estimations (dot line) of real voltage of bus 1

detection by analyzing gathered phasors.

\section{CONCLUSIONS}

This paper analyzed fault detection in power grids and proposed an enhanced WAMS architecture to improve power grid monitoring; a customized Kalman filter technique is used to analyze electric signal waveforms, using phasors representation. The Kalman filter technique has been described and applied to a dataset of 240000 phasors generated by simulating the IEEE 9-bus system behaviour. In this paper we focused on faults detection without considering their nature (e.g., malicious or accidental). The obtained results show that the Kalman filter allows to provide estimations without the heavy noise affecting the measurements. Also we highlighted that the Kalman filter shows different behaviours when in presence or absence of faults. Thus it can be used to perform fault detection.

Moving from these encouraging results, planned future work includes investigations on: i) sensitivity of the results to different values adopted in the Kalman filter for the uncertainty parameters; ii) fault detection capability of

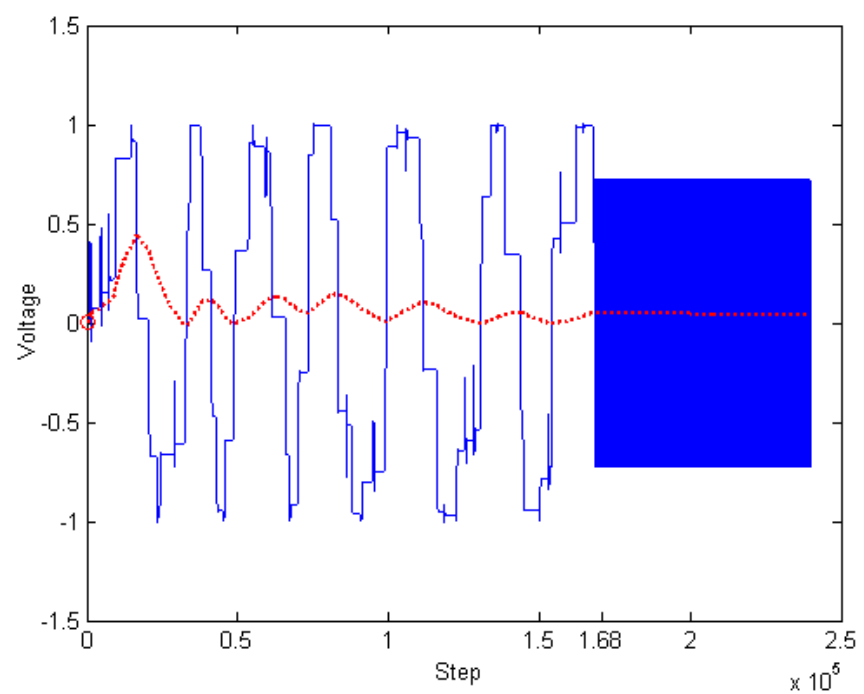

Figure 4: Measurements (solid line) and estimations (dot line) of imaginary voltage of bus 1

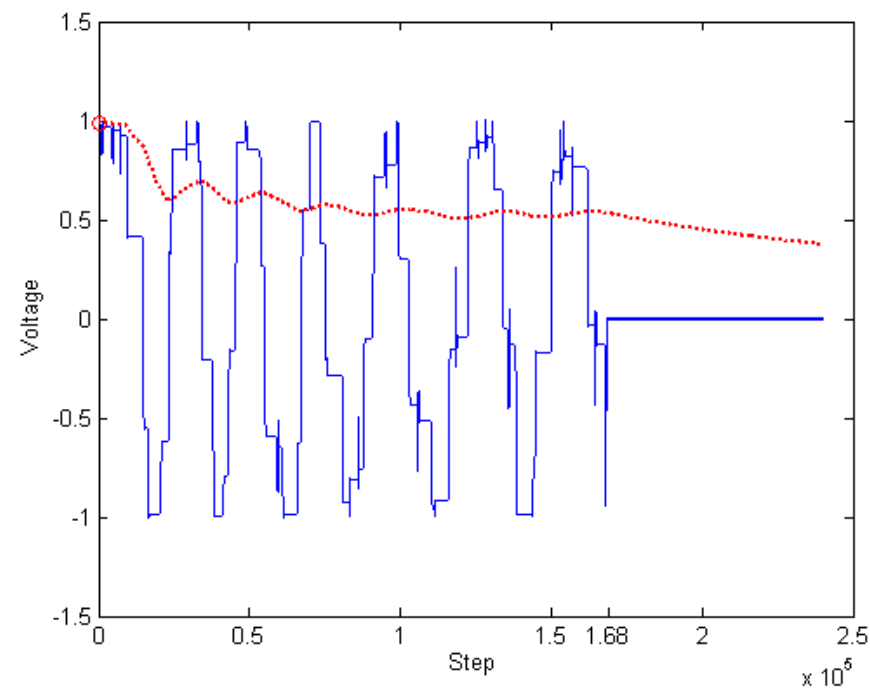

Figure 5: Measurements (solid line) and estimations (dot line) of real voltage of bus 2

Kalman filter by considering different types of faults (both accidentals and malicious); iii) effectiveness of the proposed solution based on Kalman filter with respect to other existing solutions.

\section{ACKNOWLEDGMENT}

This work has been partially supported by the TENACE PRIN Project (n. 20103P34XC) funded by the Italian Ministry of Education, University and Research and the European Project SmartC2Net (n. ICT-318023). 


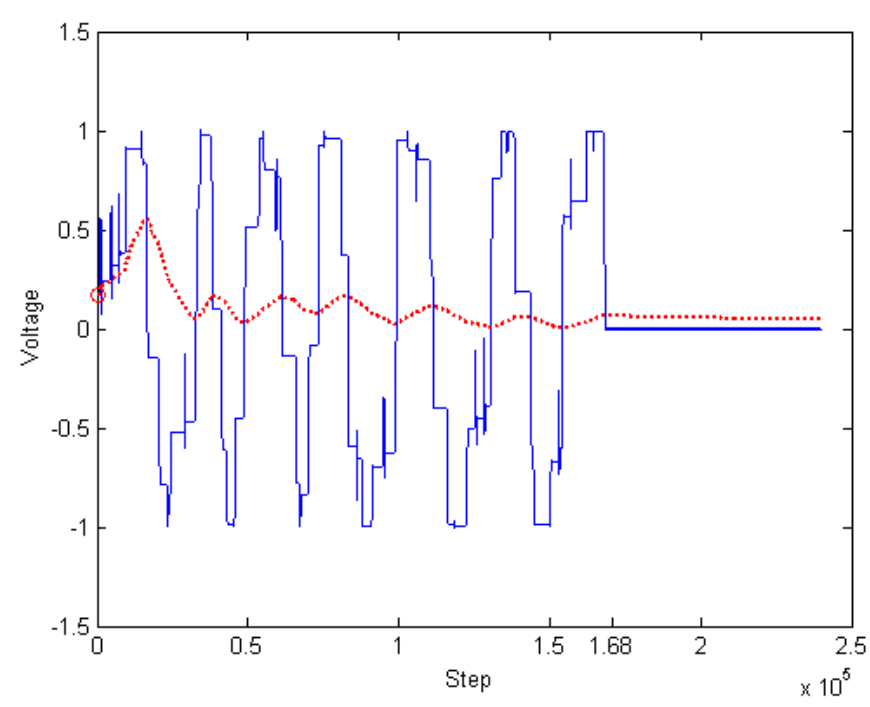

Figure 6: Measurements (solid line) and estimations (dot line) of imaginary voltage of bus 2

\section{REFERENCES}

[1] D. of Homeland Security DHS, "Critical infrastructure sectors," Tech. Rep., 2014. [Online]. Available: http: //www.dhs.gov/critical-infrastructure-sectors

[2] D. of Energy, "Smart grid overview," Tech. Rep. [Online]. Available: http://energy.gov/oe/services/ technology-development/smart-grid

[3] C. Wueest, "Targeted attacks against the energy sector," Symantec, Tech. Rep., 2014. [Online]. Available: http://www.symantec.com/content/en/us/enterprise/media/ security_response/whitepapers/targeted_attacks_against_the_ energy_sector.pdf

[4] J. J. Romero, "Blackouts illuminate India's power problems," Spectrum, IEEE, vol. 49, no. 10, pp. 11-12, Oct. 2012. [Online]. Available: http://dx.doi.org/10.1109/mspec.2012. 6309237

[5] Y. Huang, S. Werner, J. Huang, N. Kashyap, and V. Gupta, "State estimation in electric power grids: Meeting new challenges presented by the requirements of the future grid," IEEE Signal Processing Magazine, vol. 29, no. 5, pp. 33-43, 2012.

[6] T. Okon and K. Wilkosz, "Comparison of weighted-leastsquares power system state estimation in polar and rectangular coordinate systems," in Environment and Electrical Engineering (EEEIC), 2010, pp. 140-143.

[7] Y. Feng, C. Foglietta, A. Baiocco, S. Panzieri, and S. D. Wolthusen, "Malicious false data injection in hierarchical electric power grid state estimation systems," in fourth international conference on Future energy systems, 2013, pp. 183-192.

[8] T. Ma, J. Xiao, J. Xu, C. Guo, B. Yu, and S. Zhu, "Handling power system alarm cascade using a multi-level flow model," Electronics and Signal Processing Lecture Notes in Electrical Engineering, vol. 97, pp. 165-175, 2011.
[9] Q. Yang, J. Yang, W. Yu, and N. Zhang, "On a hierarchical false data injection attack on power system state estimation," in Global Telecommunications Conference (GLOBECOM 2011), 2011, pp. 1-5.

[10] "Exposing vulnerabilities in electric power grids: An experimental approach," International Journal of Critical Infrastructure Protection, vol. 7, no. 1, pp. 51 - 60, 2014.

[11] L. Coppolino, S. DAntonio, I. Elia, and L. Romano, "Security analysis of smart grid data collection technologies," in Computer Safety, Reliability, and Security, ser. Lecture Notes in Computer Science, F. Flammini, S. Bologna, and V. Vittorini, Eds. Springer Berlin Heidelberg, 2011, vol. 6894, pp. 143156.

[12] J. Kumar, J. N. Rai, and R. S. Sengar, "Effect of phasor measurement unit (pmu) on the network estimated variables," International Journal on Electrical and Power Engineering,, vol. 4, no. 1, pp. 46-51, 2013.

[13] G. Valverde and V. Terzija, "Unscented kalman filter for power system dynamic state estimation," Generation, Transmission and Distribution, IET, vol. 5, no. 1, pp. 29-37, 2011.

[14] J. Zhang, G. Welch, G. Bishop, and Z. Huang, "A two-stage kalman filtering approach for robust and real-time power systems state tracking," IEEE Transactions on Sustainable Energy, vol. 5, no. 2, pp. 629-636, 2011.

[15] Z. M. Durovic and B. D. Kovacevic, "Robust estimation with unknown noise statistics," IEEE Trans. Autom. Control, vol. 2012, no. 783843, 2012.

[16] A. Garofalo, C. Di Sarno, L. Coppolino, and S. DAntonio, "A gps spoofing resilient wams for smart grid," in Dependable Computing, ser. Lecture Notes in Computer Science, M. Vieira and J. Cunha, Eds. Springer Berlin Heidelberg, 2013, vol. 7869, pp. 134-147.

[17] R. E. Kalman, "A new approach to linear filtering and prediction problems," J. Basic Eng. Trans., vol. 82, pp. 35-45, 1960.

[18] G. E. D'Errico and N. Murru, "Fuzzy treatment of candidate outliers in measurements," Advances in Fuzzy Systems, vol. 44, no. 6, pp. 1292-1296, 1999.

[19] — - "An algorithm for concurrent estimation of timevarying quantities," Meas. Sci. Technol., vol. 23, no. 045008, 2012.

[20] P. Anderson and A. Fouad, POWER SYSTEM CONTROL AND STABILITY, 2ND ED. Wiley India Pvt. Limited, 2008. [Online]. Available: http://books.google.it/books?id= 2BXOzA34qBkC

[21] R. Zimmerman, C. Murillo-Sánchez, and R. Thomas, "Matpower: Steady-state operations, planning, and analysis tools for power systems research and education," Power Systems, IEEE Transactions on, vol. 26, no. 1, pp. 12-19, Feb 2011.

[22] S. Cole and R. Belmans, "Matdyn, a new matlab-based toolbox for power system dynamic simulation," Power Systems, IEEE Transactions on, vol. 26, no. 3, pp. 1129-1136, Aug 2011. 